\title{
Stem cells of the reproductive tract of women
}

\author{
Brad B. Swelstad ${ }^{1,2,3}$, Kamna S. Balhara ${ }^{4}$, Nikta Pashai ${ }^{5}$, Edward E. Wallach ${ }^{1,2}$, \\ Candace L. Kerr ${ }^{1,6^{*}}$ \\ ${ }^{1}$ Department of Gynecology and Obstetrics, Johns Hopkins University School of Medicine, Baltimore, USA; \\ *Corresponding Author: ckerr@som.umaryland.edu \\ ${ }^{2}$ Division of Reproductive Endocrinology, Johns Hopkins University School of Medicine, Baltimore, USA \\ ${ }^{3}$ Reproductive Care Center, Sandy and Layton, USA \\ ${ }^{4}$ Department of Emergency Medicine, Johns Hopkins University School of Medicine, Baltimore, USA \\ ${ }^{5}$ Department of Biomedical Engineering, Johns Hopkins University School of Medicine, Baltimore, USA \\ ${ }^{6}$ Department of Biochemistry and Molecular Biology, University of Maryland, Baltimore, USA
}

Received 12 January 2013; revised 11 February 2013; accepted 5 March 2013

\begin{abstract}
Research in stem cells is one of the most rapidly evolving fields of investigation in medicine today. Stem cells are defined as cells that have the capacity to both generate daughter cells identical to the cell of origin (self-renewal) and to produce progeny with more restricted, specialized potential (differentiated cells). This dual ability to self-renew and differentiate offers great promise for expanding our understanding of organ systems, elucidating disease pathophysiology, and creating therapeutic approaches to difficult diseases. The goal of this review is to offer an overview of the different types of stem cells and to provide an introduction to the applications of stem cells to the field of obstetrics and gynecology.
\end{abstract}

Keywords: Embryonic Stem Cells;

In Vitro Fertilization; Induced Pluripotent Stem Cells; Primordial Germ Cells; Germline Stem Cells;

Embryonic Germ Cells; Hematopoietic Stem Cells; Mesenchymal Stem Cells; Cancer Stem Cells; Bone Marrow Stem Cells

\section{INTRODUCTION}

\section{Overview of Stem Cells}

All stem cells have the ability to self-renew. However, they differ in their developmental potential or differentiation plasticity. For instance, totipotent cells have the ability to give rise to all cell types of the developing embryo, including embryonic and extraembryonic tissues. The fertilized egg, or zygote, at day 0 , has these characteristics. As the zygote matures into a morula and then a blastocyst at day 5, cells within the inner cell mass (ICM) of the blastocyst differentiate and lose developmental potential, giving rise to embryonic tissues comprised of the three germ layers; endoderm, ectoderm, and mesoderm. Pluripotent stem cells from the ICM or morula stage can be isolated and cultured, which have the ability to differentiate into cell types from all three embryonic germ layers. These cells are referred to as embryonic stem cells (ESCs).

Other stem cells isolated further along in embryonic development from the child or adult are more limited in their potential to differentiate along such diverse lineages. Multipotent stem cells have the ability to derive cell types from lineages other than their germ layer of origin. Mesenchymal stem cells are an example of a multipotent stem cell as they can differentiate into multiple cell types that span the ectodermal and mesodermal lineages. Other stem cells have more limited differentiation potential in that they can only differentiate into cells of a related lineage such as neural progenitor cells which can develop into both neuronal and glial cell types. Likewise, proliferative stem cells that are committed to a single lineage are unipotent. Primordial germ cells are unipotent and develop into germ cells in the adult. In contrast to stem cells, committed somatic cells are referred to as nullipotent. This group represents the most differentiated cells. It has recently become possible to reprogram somatic cells in vitro to a less differentiated state. These cells are called induced pluripotent stem cells (iPSCs).

ESCs differentiate not only into the three germ layers, but also into the germ cell lineage with the progenitors of sperm and egg which are termed primordial germ cells (PGCs). These progenitor cells have the potential to develop into pluripotent stem cells like ESCs in vivo and in vitro. In vivo, these cells are isolated from embryonal carcinomas. In vitro, culturing conditions have been developed to generate pluripotent stem cell lines from PGCs. When PGCs are isolated from late embryonic 
development they are embryonic germ cells (EGCs) and when they are isolated from the adult they are termed germline stem cells (GSCs) [1]. iPSCs have also been generated by reprogramming differentiated adult somatic cells using genetic engineering. ESCs, GSCs, and EGCs represent varying levels of pluripotency and multipotency, and together with iPSCs, are potential alternatives for studying stem cell biology and germ cell development.

\section{EMBRYONIC-LIKE STEM CELLS}

Embryonic stem cells (ESCs) are isolated from the inner cell mass of five-to-seven day-old blastocysts, and are cultured with a fibroblast feeder layer which contributes the appropriate growth factors. The ICM has generally been obtained from poor quality embryos donated from in vitro fertilization (IVF) programs that would otherwise have been discarded; this source, however, presents political, ethical, and scientific challenges. Current research on ESCs focuses on employing hypoxic conditions to improve efficiency, deriving ESCs in animal-free conditions, and using epigenetic modulators to prevent ESC differentiation in culture. Human ESCs have the potential to develop into many cell types including retinal cells, chondrocytes, cardiomyocytes, and cells of the neural lineage. Their use has been suggested as a possible approach to the treatment of a group of diseases including diabetes, heart disease, and spinal cord injury.

Embryonic germ cells (EGCs) are derived from PGCs. PGCs are present in the fetal genital ridge by the fifth week of human development, and begin mitotic expansion at around 5 weeks in the female and at 8 weeks in the male. PGCs in both sexes arrest their expansion at week 10. To derive EGCs, PGCs are isolated at their proliferative peak between 5 and 13 weeks gestation. Research is currently directed at defining culture conditions that would best facilitate derivation of EGCs. Challenges in deriving EGCs include difficulties in obtaining PGCs, in disaggregating colonies and in ensuring long-term maintenance in culture. EGCs have been used in rat models to promote repair of motor neuron injuries, cartilage degeneration and in bladder defects.

Germ-line stem cells (GSCs) are derived from testicular biopsies in men. Male testes contain spermatogonia stem cells that have the ability to both self-renew and differentiate into sperm. While research into potential clinical applications of GSCs is ongoing, these cells provide a potential adult source of pluripotent stem cells associated with fewer ethical concerns. However, the use of GSCs is limited by their uniparental epigenetic imprints and possible restriction for use only in male patients.
Induced pluripotent stem cells (iPSCs) are derived from somatic, differentiated adult cells and offer patientspecific cell based treatments as well as unprecedented in vitro human models for studying the molecular mechanisms involved in human disease. They also offer another solution to the ethical dilemma surrounding the use of ESCs. As iPSCs have the potential to generate patientspecific cell lines, their use thwarts one of the primary concerns with ESC based therapy, that of tissue rejection. As iPSCs have also been derived from many sources including fibroblasts, keratinocytes, hepatocytes, neural stem cells, and gastric epithelial cells they also provide a wide source of target-specific tissues for studying a multitude of afflictions.

More recently, studies have aimed to develop strategies for transdifferentiation, in which a cell of a specific lineage is converted to another lineage and then into another cell type without reverting back to a common progenitor state. For example, research groups have independently demonstrated the ability to reprogram B lymphocytes into macrophages as well as mature pancreatic exocrine cells into endocrine beta cells. In both reprogramming strategies, genetic integration using viruses has been employed. There are however, complications associated with the use of genetically integrated viral vectors including inflammatory response, tumorigenicity, random integration, virulence, immunogenicity, replication, and reactivation. To circumvent these issues, newer models have utilized non-integrative reprogramming methods including transfecting cells with non-integrating episomal vectors, synthetic modified mRNA, and proteins.

Since transdifferentiation involves the forced expression of genes that are specific to the desired cell type, the generation of iPSCs necessitated the use of oncogenic factors as well as other genes that regulate pluripotency. Small molecules have also been used in the generation of iPSCs, but this approach is limited since many of these chemicals cause DNA damage. One of the challenges in iPSC induction is to avoid tumorigenesis. Future research on iPSCs focuses on establishing safe and effective methods for reprogramming cells. In animal models, iPSCs have been applied in the treatment of Parkinson's disease and acute myocardial infarction, and have been implicated as possible treatments for motor neuron disease and diabetes.

\section{STEM CELLS AND THE OVARY}

One of the central tenets of reproductive medicine has been the traditional belief that mammalian females are born with a finite population of primordial follicles. This population diminishes over the course of years, eventually exhausting the germ pool and initiating menopause prior to the end of life. In recent years, however, research 
has challenged this dogma and may offer tremendous therapeutic potential for subfertile women.

A recent study by Tilly et al. shows that similar to adult mice, ovaries in reproductively-aged women posess a rare mitotically active germ cell that can proliferatein vitro and generate oocytes both in vivo and in vitro [2]. Here, ovaries in reproductive-age women contained ovarian germ cells that express cell-surface RNA helicase, Vasa, a marker of adult human oogonial stem cells. This contradicted previous dogma that suggested oogonial stem cells were not present in females after or shortly after birth. However, by using Vasa, as an ovarian stem cell marker, this study was able to provide strong evidence for the existence of proliferative female germ cell stem cells in the adult ovary which is self-renewing and is potentially able to replenish oocytes and follicle production in women. Previous studies have also postulated that ovarian neo-oogenesis occurs in adult mammalian ovaries, from possible GSC precursors capable of renewing the postnatal follicular pool. Current concepts for and against the concept of follicular renewal have been recently reviewed [3]. One of the earliest arguments supporting the idea of female GSCs was based on the observation that when the initial number of follicles at birth is combined with the incidence of follicle loss and rate of clearance of atretic follicles, the calculated number of remaining follicles in late adulthood is higher than mathematically predicted. Therefore, it was suggested that neo-oogenesis with follicle renewal plays a role in maintaining the follicle pool present in late adulthood.

However, an argument against this hypothesis is that if ovarian cells do have stem cell-like capabilities to regenerate, why does menopause occur? Nonetheless, one explanation for the development of menopause is the renewed understanding of how follicular renewal depends on the support of its environment and how the functioning hormonal and immune systems is required for germ cell maintenance. Several studies have shown the effects of aging of these supportive factors and how they may contribute to the cessation of oocyte renewal despite the continued presence of GSCs (reviewed in [4]). Thus, with increasing age there is less robust support from the stroma for stem cell survival, leading to the depletion of follicles and subsequently menopause. Similarly, decreases in autoimmunity which accompany aging have also been suggested as a contributing factor.

Evidence for postnatal follicular renewal has also been presented in several recent investigations in mice. One study showed that the follicle pool remains constant from day 12 to day 100 of postnatal life, instead of being reduced by $50 \%$ as it would have been if only follicle loss were occurring [5]. In another study, mouse ovaries exposed to doxorubicin, a drug that selectively targets oocytes, demonstrated a reduction in primordial follicle reserve; yet, once this nadir was reached, a spontaneous renewal of the follicle pool occurred within a few hours [6]. Likewise, it was also demonstrated that oogenesis could be induced in post-natal mouse ovaries via modifications to signaling pathways that govern early germ cell specification.The source of this postnatal oocyte and follicle production was suggested to come from proliferative GSCs, albeit with much debate [3,7].

Two other sources have been proposed for the origin of the GSCs that may contribute to postnatal follicular production. Some data have been presented which suggest that the ovarian surface epithelium (OSE) has the potential to differentiate into cells of the granulosa phenotype in addition to epithelial, neural, and mesenchymal cell types, and when stimulated with estrogen, it differentiates directly into oocytes. This bipotential nature of the OSE points to it as a potential endogenous ovarian source of germ cells [8,9]. In contrast, it was later suggested by the same group that the bone marrow acts as an exogenous source of ovarian germ cells. This is in accordance with multiple studies which have shown that bone marrow (BM) cells seed the ovaries after traversing the peripheral blood and that bone marrow transplant (BMT) can rescue fertility and generate immature oocytes in mice following chemotherapy-induced ovarian failure [3]. Similar models have also been applied to fertility restoration in women following chemotherapy. Successful pregnancies have been reported following BMT in several patients suffering from Fanconi's anemia or receiving high dose chemotherapy for cancer treatment [10]. The return of fertility has also been demonstrated after autologous stem cell transplantation [11]. These rare incidences have led researchers to question the evidence provided for the existence of neo-oogenesis [7]. The debate centers on whether ovarian toxic chemotherapeutic drugs reduce follicle reserve by affecting all stages of follicular development equally, since some stages of development are more resistant. While restoration of fertility in chemotherapy-exposed mice following BMT has been interpreted as supporting evidence for neo-oogenesis, opposing explanations exist for fertility restoration. Among these is the theory that chemotherapeutic regimens induce autoimmunity to ovarian antigens, and that chimerism induced by BMT allows for evasion from the autoimmune response, thereby allowing for recovery of fertility.

While the source of ovarian GSCs remains controversial, the potential applications for fertility restoration are promising. One such population includes women of child-bearing age with cancer who become infertile as a result of chemotherapy. Oocyte cryopreservation and embryo cryopreservation have been demonstrated as possible options for restoring fertility for women with low ovarian reserve, although oocyte cryopreservation 
has not yet proven to be very efficient in humans. Embryo cryopreservation has the highest success rates for future pregnancy, but may not be viable option for women without a partner or in prepubertal patients [12-14]. However, transplantation of ovarian tissue has been shown to successfully restore fertility. For example, an autologous heterotopic ovarian transplant performed in a lymphoma survivor who had been menopausal for 2.5 years due to chemotherapy, resulted in a healthy delivery at 40 weeks of gestation [15]. For women with idiopathic low ovarian reserve, incidences of donor ovarian cortical tissue between fertile and infertile monozygotic twins and genetically non-identical sisters have resulted in successful pregnancies [16,17]. The modalities of BMT, autologous ovarian cryopreservation and transplantation from donor ovaries offer hope for women with iatrogenic or pathologically-caused premature ovarian failure.

\section{STEM CELLS AND THE UTERUS}

The uterus has the ability to renew itself cyclically under hormonal influences during the menstrual cycle. It is postulated that multipotent stem cells residing in the basalis layer of the endometrium are responsible for cyclic endometrial renewal. Under systemic hormonal changes, such as rises in serum estradiol, these cells may migrate and give rise to progenitor cells that commit to specifically differentiated cell types (including epithelial, stromal, and vascular) to allow for regeneration of the endometrium [18]. A recent review published by Gargett summarizes current evidence for the existence of endometrial stem cells [19]. In 2004, Gargett's group demonstrated the existence of endometrial stem cells by showing the presence of rare epithelial and stromal cells that exhibited both clonogenicity and high proliferative potential. Using label-retaining cells (LRC) in 2006, Gargett et al. identified somatic stem cells in vivo, and demonstrated the presence of both epithelial and stromal LRC in mouse endometrium. In 2006, Chan and Gargett also demonstrated that some of these LRCs are recruited into the cell cycle by estrogen. These cells are also present in inactive and perimenopausal tissue, perhaps explaining the ability of atrophic endometrium to regenerate under estrogen replacement therapy.

The source of endometrial stem cells remains undetermined. In 2005, Snyder and Loring hypothesized that they are remnants of fetal epithelial and mesenchymal stem cells that remained in the adult endometrium after embryonic development [20]. Circulating bone marrow stem cells have also been proposed as a possible source of endometrial stem cells. Donor-derived endometrial stromal and epithelial cells were detected in women who had received bone marrow transplants (BMTs), indicating that bone marrow stem cells (BMSCs) may participate in endometrial regeneration. It is unknown whether the engraftment of BMSCs into the uterus is indeed a universally physiologic process or is restricted to BMT recipients [19].

It is possible that the causative mechanisms of diseases involving endometrial proliferation, such as endometriosis and adenomyosis, involve endometrial stem cells. Endometriosis is the growth of endometrium outside the uterine cavity. Endometriotic lesions are monoclonal and have the ability to differentiate into ovarian clear cells and endometrioid carcinomas suggesting that endometriosis could have a stem cell origin. If BMSCs are indeed involved in engrafting the endometrium and differentiating into endometrial cells, it is plausible that they may be responsible for seeding sites outside the endometrium, creating the ectopic endometrial tissue that causes endometriosis. Adenomyosis involves invasion of myometrium by basal endometrium and smooth muscle hyperplasia. It has been suggested that endometrial stem cells may abnormally migrate from the basalis to the myometrium, instead of to the functionalis layer where their progeny usually reside. Thus, pockets of endometrial tissue would be created aberrantly in the myometrium [19]. The possible involvements of BMSCs and endometrial stem cells in the pathophysiology of gynecological diseases and regeneration of endometrium offer great potential for creating therapies for disorders of the uterine endometrium.

Endometrial stem cells also offer clinical applications for general medicine. Endometrial stromal clones have shown the ability to differentiate into adipocytes, smooth muscle cells, chondrocytes and osteoblasts. It has also been shown that endometrial-derived stem cells may have a role to play in regenerating dopamine in murine Parkinson's disease models as well as in enhancing insulin production in animal models [21,22]. Recently, it was shown that stem cells from menstrual blood can provide neuroprotection in experimentally induced strokes in rats [23]. In addition to being a potential source of preexisting stem cells, endometrial tissue can also become a source of induced pluripotent stem cells. Park et al. recently demonstrated that the human endometrium expresses elevated levels of pluripotent factors such as NANOG and OCT4 that result in more efficient generation of iPSCs as compared to conventional somatic cells [24].

\section{STEM CELLS AND THE PLACENTA}

The placenta is a rich source of multiple stem cell populations. It has long been believed that hematopoiesis in the fetus was a result of the migration of embryonic yolk-sac derived hematopoietic stem cells (HSCs) to the fetal liver, and subsequently, after birth, to the final site of adult hematopoiesis, the bone marrow [25]. Subsequent research proposed that another fetal site, the aorta- 
gonad-mesonephros, was also responsible for hematopoiesis [26].

Recently it has also been shown that the placenta also plays a role in hematopoiesis. Mid-gestation mouse placenta has been shown to contain a major HSC pool. Placental HSC activity begins concomitant with the development of the aorta-gonad-mesonephros region (AGM), and prior to the presence of HSCs in the circulation or fetal liver. Placental activity contributes to the rapid growth of the HSC pool, and then decreases while liver HSCs expand. Since the placenta is upstream of the liver in fetal circulation, it is possible that the pla- centa represents a source of definitive HSCs that may seed the liver. It has been suggested that these potential de novo placental HSCs arise from the allantoic meso- derm that develops from the primitive streak [26].

The placenta has also been shown to be a potential source of mesenchymal stem cells (MSCs) and placenta-derived multipotent stem cells. Placental MSCs have been shown to differentiate into osteogenic and adipogenic lineages as well as neural progenitors that have been shown to restore locomotor activity in rat brains after hypoxic-ischemic injury [27,28]. Similarly, placenta-derived multipotent cells have also demonstrated the ability to differentiate into osteoblasts, adipocytes and neural cell types [29]. In addition, transplantation of human amniotic membrane-derived MSCs has been shown to have the potential to decrease liver fibrosis and cirrhosis following carbon tetrachloride exposure [30].

\section{FETAL STEM CELLS AND MICROCHIMERISM}

Fetal stem cells are detectable within maternal blood during pregnancy and persist within maternal organ systems for year postpartum, producing microchimerism within the maternal circulation. These cells have been shown to appear early during pregnancy [31]. They have demonstrated stem cell-like plasticity within maternal tissues. For instance, XY+ microchimeric cells in maternal tissue, most likely acquired through pregnancy, demonstrate leukocyte, hepatocyte, and epithelial markers [32]. Whether their presence is deleterious or beneficial remains to be seen. Microchimeric cells have been implicated in rheumatoid arthritis remission during pregnancy [33]. They may play a role in tissue repair: fetal-derived cells have been shown to proliferate in injured organs in parous rats [34]. However, potential harm from fetal microchimeric cells seems equally great. It has been hypothesized that fetal microchimeric cells play a role in triggering systemic sclerosis after childbearing. Women with lung cancer who recently had given birth to sons were found to have male cells concentrated within their tumors rather than in normal lung parenchyma [35].
However, it is unclear if these cells act as cancer stem cells (CSCs) or are found at those sites because they participate in repair of inflamed or abnormal tissue. In some studies, fetal cells have been associated with poorer prognosis in women diagnosed with pregnancy-associated breast carcinoma [36]. Mice models also showed that higher-grade breast cancers during pregnancy contained increased incidence of fetal cells [37]. In contrast, other studies have suggested that fetal cells may be protective against cancer [38].

\section{CANCER STEM CELLS}

Tumors contain a heterogeneous group of cells, of which only a few demonstrate the ability for growth and metastasis through self-renewal and enormous proliferative potential [39,40]. These stem-cell-like pools referred to as CSCs may explain many features of cancer, including clonality and heterogeneity [41]. Cancer stem cells have been shown to play a role in acute myeloid leukemia, breast cancer and glioblastoma [39]. While more differentiated cancer cells are killed by chemotherapy, CSCs act as chemo-resistant precursors that replenish the pool. It is likely that cancer stem cells arise from normal stem cells that have accumulated changes over time allowing them to escape regulatory mechanisms and proliferate unchecked, gaining a selective advantage for clonal expansion [41]. A recent study has also demonstrated that a CSC population is responsible for the production of endometrial carcinoma tumor cells [42]. Such findings will have important implications for therapeutic approaches to cancer, such as gene therapy.

\section{CONCLUSION}

While research on stem cells continues to evolve and many topics discussed here remain unresolved, there is no doubt that stem cells hold great promise in reproducetive medicine. Stem cells could provide a mechanism for explaining reproductive pathology, for both understanding and treating cancer, and for restoring fertility in women with premature ovarian failure. The female reproductive tract also serves as a valuable stem cell source for systemic therapies. A better understanding of stem cells in the reproductive tract offers a means for enhanced comprehension of female reproductive physiology, provides novel approaches to the pathophysiology of disease and malignancy, and opens new avenues for exploring therapies for disease processes.

\section{REFERENCES}

[1] Swelstad, B.B. and Kerr, C.L. (2009) Current protocols in the generation of pluripotent stem cells: Theoretical methodological and clinical considerations. Stem Cell and 
Cloning: Advances and Applications, 2010, 13-27.

[2] White, Y.A., et al. (2012) Oocyte formation by mitotically active germ cells purified from ovaries of reproductive-age women. Nature Medicine, 18, 413-421. doi:10.1038/nm.2669

[3] Tilly, J.L., Niikura, Y. and Rueda, B.R. (2009) The current status of evidence for and against postnatal oogenesis in mammals: A case of ovarian optimism versus pessimism? Biology of Reproduction, 80, 2-12. doi:10.1095/biolreprod.108.069088

[4] Bukovsky, A. (2011) How can female germline stem cells contribute to the physiological neo-oogenesis in mammals and why menopause occurs? Microscopy and Microanalysis, 17, 498-505. doi:10.1017/S143192761000036X

[5] Kerr, J.B., et al. (2006) Quantification of healthy follicles in the neonatal and adult mouse ovary: Evidence for maintenance of primordial follicle supply. Reproduction, 132, 95-109. doi:10.1530/rep.1.01128

[6] Johnson, J., et al. (2005) Oocyte generation in adult mammalian ovaries by putative germ cells in bone marrow and peripheral blood. Cell, 122, 303-315. doi:10.1016/j.cell.2005.06.031

[7] Notarianni, E. (2011) Reinterpretation of evidence advanced for neo-oogenesis in mammals, in terms of a finite oocyte reserve. Journal of Ovarian Research, 4, 1. doi:10.1186/1757-2215-4-1

[8] Bukovsky, A., Svetlikova, M. and Caudle, M.R. (2005) Oogenesis in cultures derived from adult human ovaries. Reproductive Biology and Endocrinology, 3, 17. doi:10.1186/1477-7827-3-17

[9] Parte, S., et al. (2011) Detection, characterization, and spontaneous differentiation in vitro of very small embryonic-like putative stem cells in adult mammalian ovary. Stem Cells and Development, 20, 1451-1464. doi:10.1089/scd.2010.0461

[10] Sanders, J.E., et al. (1996) Pregnancies following highdose cyclophosphamide with or without high-dose busulfan or total-body irradiation and bone marrow transplantation. Blood, 87, 3045-3052.

[11] Hershlag, A. and Schuster, M.W. (2002) Return of fertility after autologous stem cell transplantation. Fertility and Sterility, 77, 419-421. doi:10.1016/S0015-0282(01)02987-9

[12] Al-Hasani, S., et al. (1987) Cryopreservation of human oocytes. Human Reproduction, 2, 695-700.

[13] Eroglu, A., Toth, T.L. and Toner, M. (1998) Alterations of the cytoskeleton and polyploidy induced by cryopreservation of metaphase II mouse oocytes. Fertility and Sterility, 69, 944-957. doi:10.1016/S0015-0282(98)00030-2

[14] Yoon, T.K., et al. (2000) Pregnancy and delivery of healthy infants developed from vitrified oocytes in a stimulated in vitro fertilization-embryo transfer program. Fertility and Sterility, 74, 180-181. doi:10.1016/S0015-0282(00)00572-0

[15] Oktay, K. (2006) Spontaneous conceptions and live birth after heterotopic ovarian transplantation: Is there a germline stem cell connection? Human Reproduction, 21, 1345-

\section{8. doi:10.1093/humrep/del007}

[16] Silber, S.J., et al. (2008) A series of monozygotic twins discordant for ovarian failure: Ovary transplantation (cortical versus microvascular) and cryopreservation. Human Reproduction, 23, 1531-1537. doi:10.1093/humrep/den032

[17] Donnez, J., et al. (2007) Allograft of ovarian cortex between two genetically non-identical sisters: Case report. Human Reproduction, 22, 2653-2659. doi:10.1093/humrep/dem211

[18] Padykula, H.A. (1991) Regeneration in the primate uterus: The role of stem cells. Annals of the New York Academy of Sciences, 622, 47-56. doi:10.1111/j.1749-6632.1991.tb37849.x

[19] Gargett, C.E. (2007) Uterine stem cells: What is the evidence? Human Reproduction Update, 13, 87-101. doi:10.1093/humupd/dml045

[20] Snyder, E.Y. and Loring, J.F. (2005) A role for stem cell biology in the physiological and pathological aspects of aging. Journal of American Geriatrics Society, 53, S287S291. doi:10.1111/j.1532-5415.2005.53491.x

[21] Wolff, E.F., et al. (2011) Endometrial stem cell transplantation restores dopamine production in a Parkinson's disease model. Journal of Cellular and Molecular Medicine, 15, 747-755. doi:10.1111/j.1582-4934.2010.01068.x

[22] Li, H.Y., et al. (2010) Induction of insulin-producing cells derived from endometrial mesenchymal stem-like cells. Journal of Pharmacology and Experimental Therapeutics, 335, 817-829. doi:10.1124/jpet.110.169284

[23] Borlongan, C.V., et al. (2010) Menstrual blood cells display stem cell-like phenotypic markers and exert neuroprotection following transplantation in experimental stroke. Stem Cells and Development, 19, 439-452. doi:10.1089/scd.2009.0340

[24] Park, J.H., et al. (2011) Human endometrial cells express elevated levels of pluripotent factors and are more amenable to reprogramming into induced pluripotent stem cells. Endocrinology, 152, 1080-1089. doi:10.1210/en.2010-1072

[25] McGrath, K. and Palis, J. (2008) Ontogeny of erythropoiesis in the mammalian embryo. Current Topics in Developmental Biology, 82, 1-22. doi:10.1016/S0070-2153(07)00001-4

[26] Mikkola, H.K., et al. (2005) Placenta as a site for hematopoietic stem cell development. Experimental Hematology, 33, 1048-1054. doi:10.1016/j.exphem.2005.06.011

[27] Fukuchi, Y., et al. (2004) Human placenta-derived cells have mesenchymal stem/progenitor cell potential. Stem Cells, 22, 649-658. doi:10.1634/stemcells.22-5-649

[28] Park, S., et al. (2011) Neural progenitors generated from the mesenchymal stem cells of first-trimester human placenta matured in the hypoxic-ischemic rat brain and mediated restoration of locomotor activity. Placenta, 32, 269-276. doi:10.1016/j.placenta.2010.12.027

[29] Yen, B.L., et al. (2008) Placenta-derived multipotent cells differentiate into neuronal and glial cells in vitro. Tissue Engineering Part A, 14, 9-17. doi:10.1089/ten.a.2006.0352 
[30] Zhang, D., Jiang, M. and Miao, D. (2011) Transplanted human amniotic membrane-derived mesenchymal stem cells ameliorate carbon tetrachloride-induced liver cirrhosis in mouse. PLoS One, 6, Article ID: e16789. doi:10.1371/journal.pone.0016789

[31] Lee, E.S., et al. (2010) Fetal stem cell microchimerism: Natural-born healers or killers? Molecular Human Reproduction, 16, 869-878. doi:10.1117/2.1201002.002651

[32] Khosrotehrani, K., et al. (2004) Transfer of fetal cells with multilineage potential to maternal tissue. JAMA, 292, 75-80. doi:10.1001/jama.292.1.75

[33] Guthrie, K.A., et al. (2010) Does pregnancy provide vaccine-like protection against rheumatoid arthritis? Arthritis and Rheumatism, 62, 1842-1848.

[34] Wang, Y., et al. (2004) Fetal cells in mother rat contribute to the remodeling of liver and kidney after injury. Biochemical and Biophysical Research Communications, 325, 961-967. doi:10.1016/j.bbrc.2004.10.105

[35] O’Donoghue, K., et al. (2008) Microchimeric fetal cells cluster at sites of tissue injury in lung decades after pregnancy. Reproductive Biomedicine Online, 16, 382-390. doi:10.1016/S1472-6483(10)60600-1

[36] Dubernard, G., et al. (2008) Breast cancer stroma frequ- ently recruits fetal derived cells during pregnancy. Breast Cancer Research, 10, R14. doi:10.1186/bcr1860

[37] Dubernard, G., et al. (2009) Increased fetal cell microchimerism in high grade breast carcinomas occurring during pregnancy. International Journal of Cancer, 124, 1054-1059. doi:10.1002/ijc.24036

[38] Gadi, V.K., et al. (2008) Case-control study of fetal micro-chimerism and breast cancer. PLoS One, 3, Article ID: e1706.

[39] Pardal, R., Clarke, M.F. and Morrison, S.J. (2003) Applying the principles of stem-cell biology to cancer. $\mathrm{Na}$ ture Reviews Cancer, 3, 895-902. doi:10.1038/nrc1232

[40] Clarke, M.F. and Fuller, M. (2006) Stem cells and cancer: Two faces of eve. Cell, 124, 1111-1115. doi:10.1016/j.cell.2006.03.011

[41] Miller, S.J., Lavker, R.M. and Sun, T.T. (2005) Interpreting epithelial cancer biology in the context of stem cells: Tumor properties and therapeutic implications. Biochimica et Biophysica Acta, 1756, 25-52.

[42] Hubbard, S.A., et al. (2009) Evidence for cancer stem cells in human endometrial carcinoma. Cancer Research, 69, 8241-8248. doi:10.1158/0008-5472.CAN-08-4808 